\title{
Isolated single coronary artery from noncoronary sinus.
}

\author{
Meenu Bagarhatta $^{1}$, Ritu Agarwal ${ }^{1}$, and Rengarajan Rajagopal ${ }^{1}$ \\ ${ }^{1}$ SMS Medical College and Hospital
}

September 10, 2020

\begin{abstract}
We present the CT angiogram images of a 43 year old lady with an isolated single coronary artery arising from the noncoronary sinus with a narrow slit like orifice and oblique take off.
\end{abstract}

\section{Legend:}

A "single" coronary artery arises from the aorta by a single ostium and supplies entire heart. They are extremely rare abnormalities (prevalence of less than $0.06 \%$ in angiographic studies) seen usually in association with other congenital cardiac anomalies like coronary fistulae and bicuspid aortic valve. CT coronary angiogram of a 43 year lady with atypical chest pain (VRT and axial scans - Image.1) shows a single coronary artery arising from the noncoronary sinus with a narrow slit-like and oblique take off, coursing in the anterior interventricular groove to divide into left anterior descending artery(LAD) and left circumflex artery(LCx). The conal artery $(\mathrm{CA})$ originates from mid LAD and has a pre-pulmonic course to reform a hypoplastic proximal right coronary artery $(\mathrm{RCA})$ seen in the right atrio-ventricular groove. The $\mathrm{LCx}$ is seen to give rise to a large posterior descending artery supplying the inferior surfaces of both ventricles. The left and right coronary sinuses (LCS \& RCS) are blind ending. This pattern can be classified as "IIa" course according to the Lipton classification [1]. No other intracardiac defects were seen. Patients may be asymptomatic, have non-specific symptoms, chest pain, congestive heart failure and in extreme cases, sudden cardiac death. Coronary CT angiography can precisely delineate the course of the anomalous artery and its relation to other cardiovascular structures to help planning for surgery in symptomatic patients. There is no clear consensus on management of asymptomatic patients, however, symptomatic patients and patients having a malignant course may require corrective surgical procedures for symptom relief. Our patient also had a single coronary artery with a slit like orifice and oblique take off in the absence of other congenital cardiac anomalies, which to our knowledge has not been previously reported in literature. She is under close clinical observation with a plan for bypass surgery in the event of any complications.

References:

Lipton MJ, Barry WH, Obrez I, Silverman JF, Wexler L. Isolated single coronary artery: diagnosis, angiographic classification, and clinical significance. Radiology (1979), 130(1), 39-47.

Conflicts of interest: The authors declare no conflicts of interest

Informed consent: Obtained

Funding source: None

Word count: 304 words

Acknowledgements: Nil

Image: 

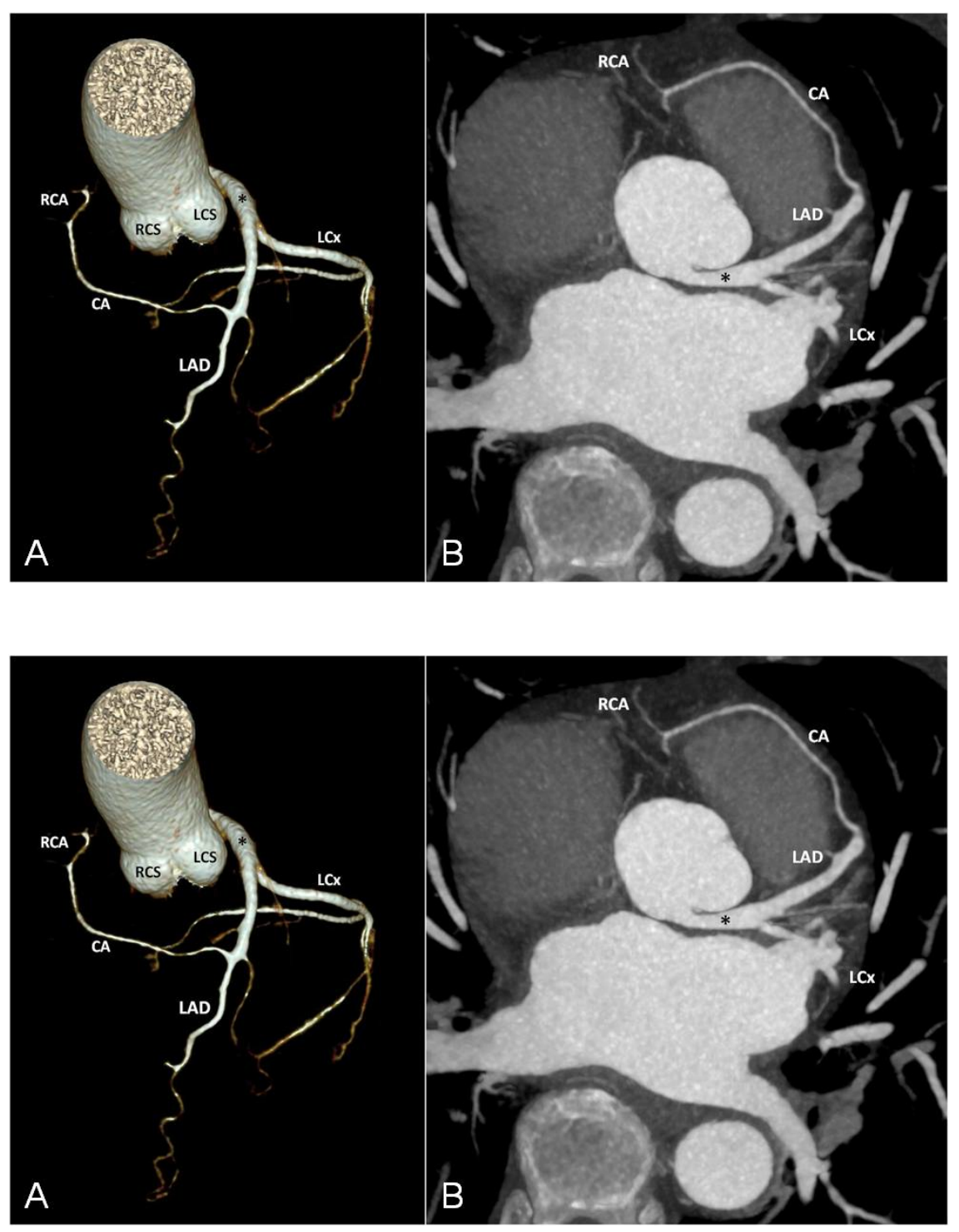